\title{
Contribution à la connaissance des Ascalaphes du Mali et description de la femelle de Tytomyia flinti Tjeder, 1992 (Neuroptera, Ascalaphidae) \\ Bruno Michel
}

\begin{abstract}
Contribution to the knowledge of the owl-flies of Mali including the description of the female of Tytomyia flinti Tjeder, 1992 (Neuroptera, Ascalaphidae). Four species of owl-flies, Allocormodes maculipennis Taschenberg, 1879, Phalascusa pardalis (Gerstaecker, 1888), Cirrops kumari Tjerder, 1980 and Tytomyia flinti Tjeder, 1992 are recorded for first time from Mali. The resting positions of $\mathrm{C}$. kumari and $T$. flinti are presented. The female of $T$. flinti is described. The records of Farakosius chloeae Michel, 1998 and $F$. thaisae Michel, 1998 since their description are indicated. The flight period of the species was determined and some information on their bio-ecology is given.
\end{abstract}

\section{Résumé}

Quatre espèces d'ascalaphes, Allocormodes maculipennis Taschenberg, 1879, Phalascusa pardalis (Gerstaecker, 1888), Cirrops kumari Tjerder, 1980 et Tytomyia flinti Tjeder, 1992, sont signalées pour la première fois du Mali. Les attitudes de repos de $C$. kumari et $T$. flinti sont présentées. La femelle de T. flinti est décrite. Les captures de Farakosius chloeae Michel, 1998 et F. thaisae Michel, 1998, postérieures à leur description sont indiquées. Les périodes de vol des espèces sont précisées et des informations sur leur bio-écologie sont apportées.

\section{Citer ce document / Cite this document :}

Michel Bruno. Contribution à la connaissance des Ascalaphes du Mali et description de la femelle de Tytomyia flinti Tjeder, 1992 (Neuroptera, Ascalaphidae). In: Bulletin de la Société entomologique de France, volume 105 (1), mars 2000. pp. 53-59; https://www.persee.fr/doc/bsef_0037-928x_2000_num_105_1_16640

\section{Ressources associées:}

Tytomyia flinti

Fichier pdf généré le 30/09/2019 


\title{
Contribution à la connaissance des Ascalaphes du Mali et description de la femelle de Tytomyia flinti Tjeder, 1992 (Neuroptera, Ascalaphidae)
}

\author{
par Bruno MICHEL \\ DORAS Center, c/o International Affairs Relation, Kasetsart University, Bangkhen, 10900 Bangkok, Thaïlande
}

Résumé. - Quatre espèces d'ascalaphes, Allocormodes maculipennis Taschenberg, 1879, Phalascusa pardalis (Gerstaecker, 1888), Cirrops kumari Tjerder, 1980 et Tytomyia flinti Tjeder, 1992, sont signalées pour la première fois du Mali. Les attitudes de repos de $C$. kumari et $T$. flinti sont présentées. La femelle de $T$. flinti est décrite. Les captures de Farakosius chloeae Michel, 1998 et $F$. thaisae Michel, 1998, postérieures à leur description sont indiquées. Les périodes de vol des espèces sont précisées et des informations sur leur bio-écologie sont apportées.

Summary. - Contribution to the knowledge of the owl-flies of Mali including the description of the female of Tytomyia flinti Tjeder, 1992 (Neuroptera, Ascalaphidae). Four species of owl-flies, Ailocormodes maculipennis Taschenberg, 1879, Phalascusa pardalis (Gerstaecker, 1888), Cirrops kumari Tjerder, 1980 and Tytomyia flinti Tjeder, 1992 are recorded for first time from Mali. The resting positions of $C$. kumari and $T$. flinti are presented. The female of $T$. flinti is described. The records of Farakosius chloeae Michel, 1998 and $F$. thaisae Michel, 1998 since their description are indicated. The flight period of the species was determined and some information on their bio-ecology is given.

Mots clés. - Neuroptera, Ascalaphidae, bio-écologie, région afrotropicale, Mali.

Les Ascalaphes afrotropicaux sont mal connus et seules quelques tribus ont fait l'objet de révisions récentes (TJEDER, 1992; TJEDER \& HANSSON, 1992). Ces travaux concernent surtout les espèces australes qui sont à présent assez bien répertoriées. La faune de l'Afrique de l'Ouest par contre demeure peu étudiée et un grand nombre d'espèces ne sont pas identifiables. Pour le Mali très peu d'informations sont disponibles. Un seul Ascalaphe, Helicomitus festivus (Rambur, 1842) (= Ascalaphus festivus), provenant du delta intérieur du Niger, est mentionné dans la littérature que nous avons consultée (FRASER, 1953). D'après TJEDER (1992) aucune espèce n'est connue du sud du pays.

De 1993 à 1998 nous avons récolté de nombreux Ascalaphidae et Myrmeleontidae dans le sud du Mali, en particulier dans la région de Sikasso, située dans l'extrême sud-est du pays. Certains résultats ont déjà été publiés et les stations de récolte ont été décrites (MiCHEL, 1998, 1999). Dans cette troisième note nous présentons les données relatives aux Ascalaphes que nous avons pu identifier, et nous apportons des précisions sur les espèces nouvellement décrites (MICHEL, 1998).

\section{LISTE COMMENTÉE DES ESPÈCES}

\section{HAPLOGLENIINAE}

1 - Allocormodes maculipennis Taschenberg, 1879 (fig. 1).

Stations et dates de récolte. - Ville de Sikasso $\left(11^{\circ} 19^{\prime} \mathrm{N}-05^{\circ} 41^{\prime} \mathrm{W}\right): 28 . \mathrm{V} .95,19$, capturée le matin, posée contre un mur au-dessous d'une lampe. Farako $\left(11^{\circ} 12^{\prime} \mathrm{N}-05^{\circ} 29^{\prime} \mathrm{W}\right): 2 . \mathrm{IV} .97,20 \mathrm{~h} 30$, 19 , piège lumineux.

Capturé en avril - mai, au début de la saison des pluies. Les deux femelles ont été attirées par la lumière. 
Espèce identifiée à partir de la photographie d'un exemplaire conservé au Muséum national d'Histoire naturelle, à Paris, que nous devons à la courtoisie du Dr A. Prost. L'autre espèce présente en Afrique de l'Ouest, Allocormodes intractabilis (Walker, 1860), se rencontre en zone plus humide. Nous l'avons récoltée à Bouaké (Côte d'Ivoire).

\section{ASCALAPHinAE}

\section{2 - Phalascusa pardalis (Gerstaecker, 1888) (fig. 2)}

Stations et dates de récolte. - Ville de Sikasso: 14.XI.96, 1 \% Sikasso (prairies à graminées en bordure de la piste d'aviation): 27.X.95, 1 ơ. Prairie à graminées à $1 \mathrm{~km}$ au nord de Sikasso: 8.X.97, $10 ; 13 . X .97,1$ \%. Prairies à graminées en bordure de la piste de Longorola à environ $11 \mathrm{~km}$ au nord de Sikasso: 30.X.95, 3o 13-XI-95, 19; 21.XI.97, 19. Chutes de Farako: 28.X.96, vers 16 h, 19 Natiékoura $\left(11^{\circ} 20^{\prime} \mathrm{N}-05^{\circ} 51^{\prime} \mathrm{W}\right): 26 . \mathrm{X} .95,20^{\circ}, 7{ }^{\circ}$. Dandéresso $\left(11^{\circ} 29^{\prime} \mathrm{N}-05^{\circ} 29^{\prime} \mathrm{W}\right): 4 . \mathrm{X} .96$, 1 . Foulabala, région de Manankoro $\left(10^{\circ} 40^{\prime} \mathrm{N}-07^{\circ} 22^{\prime} \mathrm{W}\right): 25 . \mathrm{X} .96,29$. Kadiolo $\left(10^{\circ} 32^{\prime} \mathrm{N}-05^{\circ}\right.$ $\left.44^{\prime} \mathrm{W}\right): 22 . \mathrm{X} .96$, vers $15 \mathrm{~h}, 10^{\circ}, 1$. Djidjan, région de Kita $\left(13^{\circ} 12^{\prime} \mathrm{N}-09^{\circ} 26^{\prime} \mathrm{W}\right): 26.1 \mathrm{X} .96,19$.

Capturé du 26 septembre au 21 novembre. Vole au début de la saison sèche. Très commun dans les formations à graminées.

Espèce à vaste répartition: Sénégal, Gambie, Guinée, Sierra Léone, Nigéria, République Centrafricaine, Zaïre, Uganda (TJEDER, 1980).

\section{3 - Cirrops kumari Tjeder, 1980}

Stations et dates de récolte. - Prairie à graminées à $1 \mathrm{~km}$ au nord de Sikasso: 12.XI.96, 1 ․ Prairies à graminées en bordure de la piste de Longorola à environ $11 \mathrm{~km}$ au nord de Sikasso: 30.X.95, $1 \delta^{\dagger} ; 31 . X .95,16 \mathrm{~h}, 2 q ; 5 . X I .96$, vers $17 \mathrm{~h}, 2 \% ; 13 . X I .95$, entre $15 \mathrm{~h} 30$ et $17 \mathrm{~h}, 9 \delta, 8 q$ ( $1 \delta^{\circ}$ et $1 q$

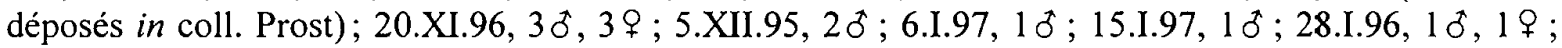
29.I.96, $1 \delta^{\star}, 1 q ; 30 . I .96,1 \delta^{\star}, 1 \%$; 7.II.97, $1 \delta^{\circ} ; 24.11 .96,1$ \%. Pégnasso $\left(11^{\circ} 28^{\prime} \mathrm{N}-05^{\circ} 50^{\prime} \mathrm{W}\right)$ : 5.XI.96, vers 17 h, 2 q. Natiékoura: 4.XI.97, 1 ; 6.XI.96, 16 h 30, 1 ; 15.XI.96, 1 o ; 22.XI.95, $1 \delta^{\circ}$; 30.I.96, $2 q ; 2 . I I .98$, entre 16 h et 17 h, $28,1 \% ; 16 . I I .96,1 q ; 27 . I I .97,19$. Chutes de Farako:

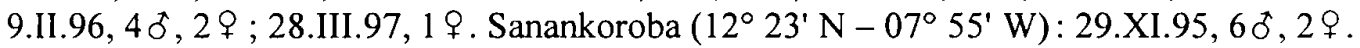

Capturé du 30 octobre au 28 mars. Vole pendant la saison sèche. Les premiers adultes apparaissent peu de temps après la fin de la saison des pluies.

Cette espèce était connue de Gambie (4 exemplaires), du Ghana ( 1 ex.) (TJEDER, 1980) et du Nigéria (3 ex.) (HYND, 1992). A. Prost, qui a chassé pendant une dizaine d'années surtout au Burkina Faso, mais également dans toute la sous-région, ne l'a jamais capturée (Prost, comm. pers.). Elle est très commune dans le sud du Mali dans les prairies à graminées et les savanes arborées mais présente, semble-t-il, une distribution localisée.

Au cours de la journée, elle se tient sur les tiges des plantes sèches ou les petites branches des arbustes à une hauteur comprise le plus souvent entre $50 \mathrm{~cm}$ et $1,5 \mathrm{~m}$. En fin d'après-midi elle se laisse facilement capturer à la main. Les adultes adoptent différentes positions de repos, variables selon le sexe. Les femelles maintiennent leur abdomen à $80^{\circ}$ par rapport au support (fig. 3). Les mâles se posent soit en gardant le thorax et l'abdomen dans le prolongement l'un de l'autre, le corps faisant un angle de $34^{\circ}$ avec le végétal (fig. 5), soit en relevant l'abdomen à $60^{\circ}$ (fig. 4). Cette dernière position pourrait correspondre à l'émission de phéromones (Mansell, comm. pers.). Chez les deux sexes, la face est appliquée contre le support, les antennes sont dressées et les massues de forme aplatie sont dirigées en arrière. Les ailes sont repliées vers le bas (fig. 3 à 5).

Une femelle, capturée le 20.XI.96, a l'antenne gauche enroulée en spirale (fig. 6).

\section{4 - Tytomyia flinti Tjeder, 1992}

Stations et dates de récolte. - Prairies en bordure de la piste de Longorola (environ $11 \mathrm{~km}$ au nord de Sikasso): 30.I.96, $20^{\dagger} ;$ 5.II.98, $2 \delta^{\dagger} ;$ 7.II.97, $2 \delta^{\circ}, 1 q ; 22 . I I .96,29 ; 23.11 .96,1 \% ; 24 . I I .96$, 

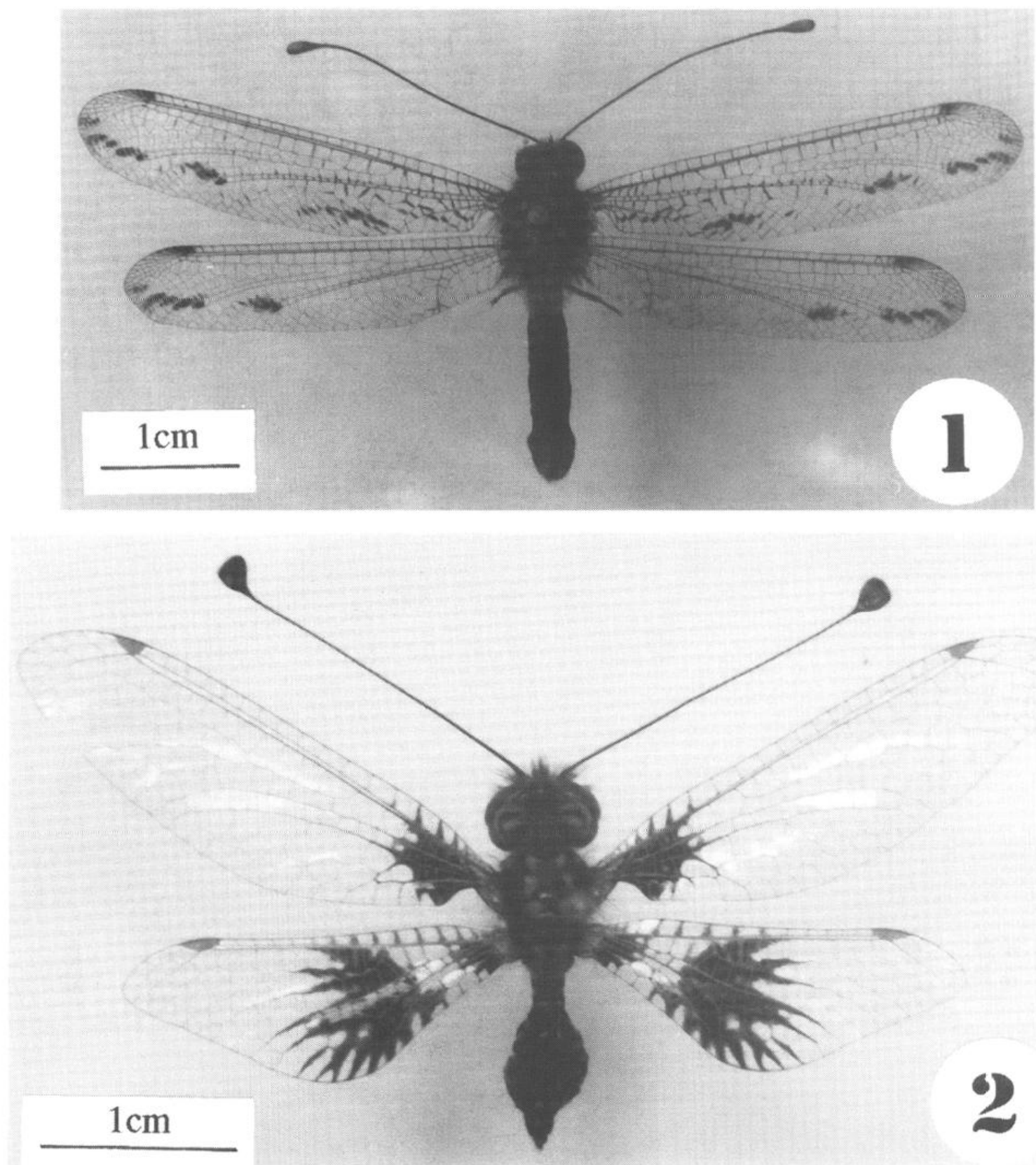

Fig. 1 et 2. -1 , Allocormodes maculipennis Taschenberg, $९ .-2$, Phalascusa pardalis (Gerstaecker), $\uparrow$.

$1 q ; 28.11 .96,2 q ; 1 . I I I .96,1 q ; 5 . I I I .96,10,4 q ; 6 . I I I .96,20,3 q$ ( 1 \% et $1 q$ déposés in coll. Prost); 9.III.96, vers 11 h $30,1 q ; 10 . I I I .97,16$ h $30,1 \delta^{\star}, 1 q ; 11 . I I I .96$, vers 11 h $30,1 q ; 14 . I I I .96,1 \delta^{\circ}, 19$; 1.IV.96, $19 ; 5 . I V .96,19$. Pégnasso: 10.IV.97, $19 ; 25 . I V .98,12$ h, $19 ; 11$. V.97, 1 \%. Natiékoura: 2.II.98, vers 16 h $30,2 \delta ; 10 . I I .97,17$ h, $1 \delta^{*} ; 16 . I I .96,19 ; 22 . I I .97,1 \% ; 24 . I I .97$, vers $17 \mathrm{~h}, 19$; 4.III.98, vers $17 \mathrm{~h}, 10$, $2 q ; 23$. III.98, $19 ; 31 . I I I .96,11 \mathrm{~h}, 1 q$. Chutes de Farako: 7.III.98, vers $11 \mathrm{~h}$, $1 \% ; 16 . I I I .97,20 \mathrm{~h}$, à la lumière, $1 \delta^{\circ}$. Mamouroubougou (près des chutes de Farako): 12.I1I.96, 1 \% . Environs de $\operatorname{San}\left(13^{\circ} 17^{\prime} \mathrm{N}-04^{\circ} 56^{\prime} \mathrm{W}\right)$ : 12.V.97, vers $16 \mathrm{~h}, 4 \delta^{\circ}, 2$..

Capturé du 30.I au 12.V, du milieu de la saison sèche jusqu'au début de la saison des pluies. Cette espèce a été décrite par TJEDER (1992) à partir de deux mâles, l'un du Sénégal, l'autre du Burkina Faso. La face sternale de l'abdomen de certains mâles présente une coloration bleu clair. Ce changement chromatique pourrait correspondre à un caractère sexuel secondaire. Il s'observe sur le vivant et disparaît après séchage. La femelle n'avait jamais été capturée. 


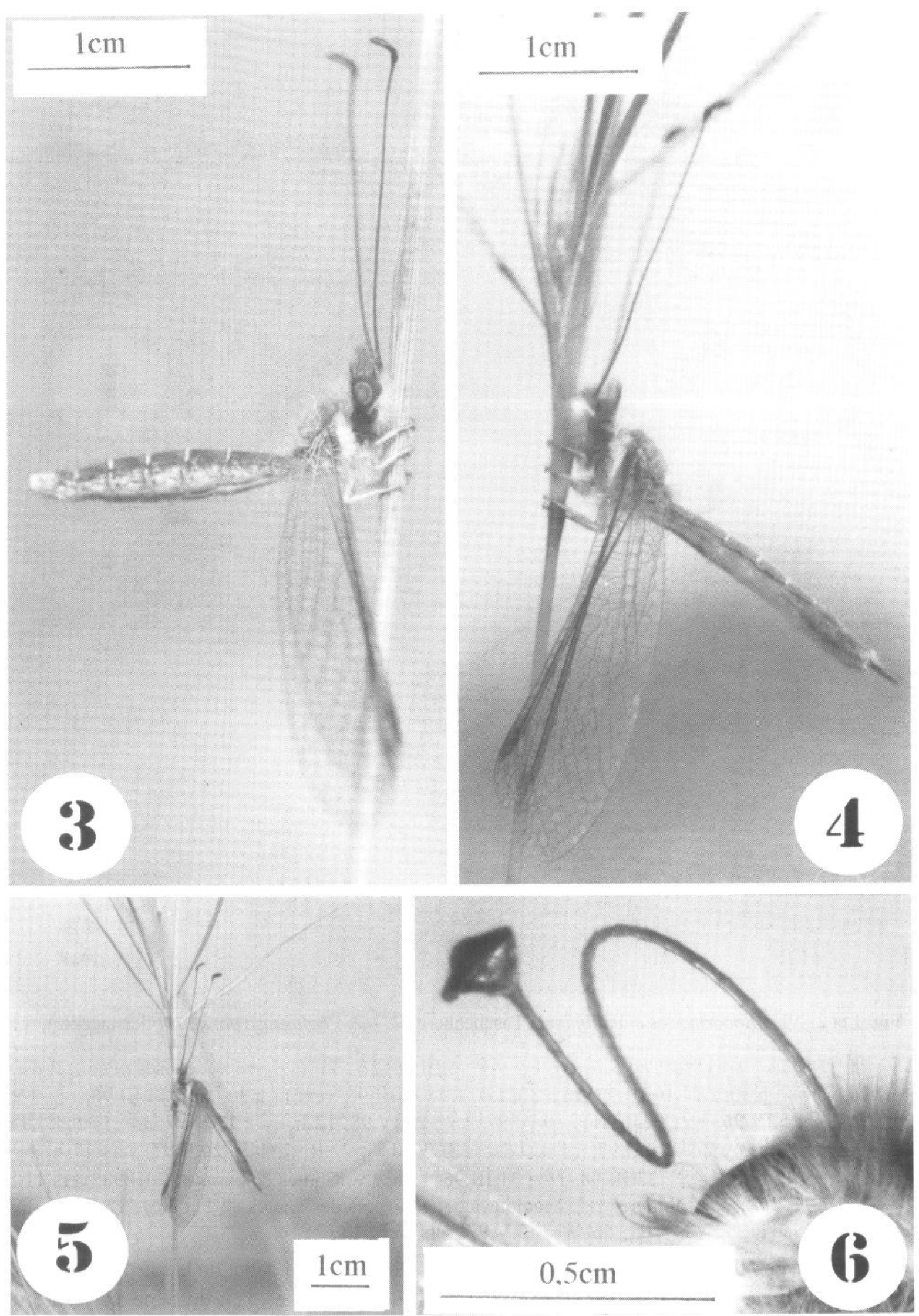

Fig. 3 à 6 . - Cirrops kumari Tjeder. -3.9 au repos. - 4, 5, o au repos, abdomen relevé et baissé. - 6, Antenne gauche mal formée. 

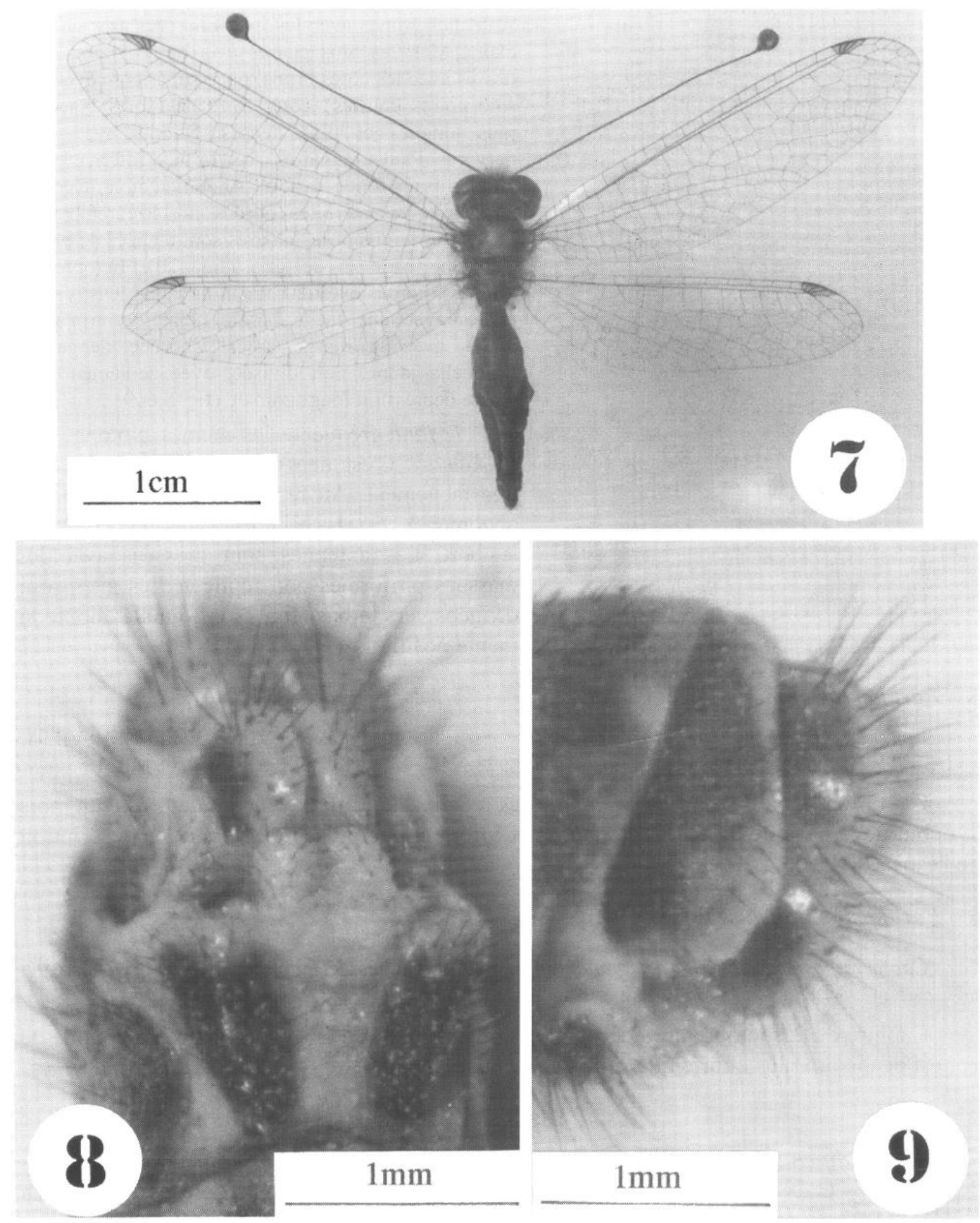

Fig. 7 à 9. - Tytomyia flinti Tjeder, $९$, habitus et extrémité abdominale, vues ventrale et latérale gauche.

Description de la femelle (fig. 7).

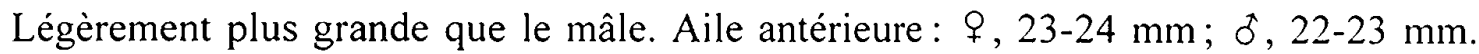

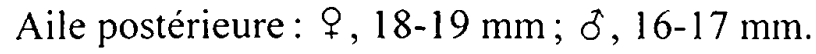

Coloration identique à celle du mâle. Vertex et front brun foncé avec des soies grises associées à des soies noires sur le vertex. Joues blanches largement bordées de noir le long du front. Pleurostomes, labre et clypéus jaunâtres. Sclérite post-orbital blanchâtre. Occiput brun avec une tache jaune latérale. 


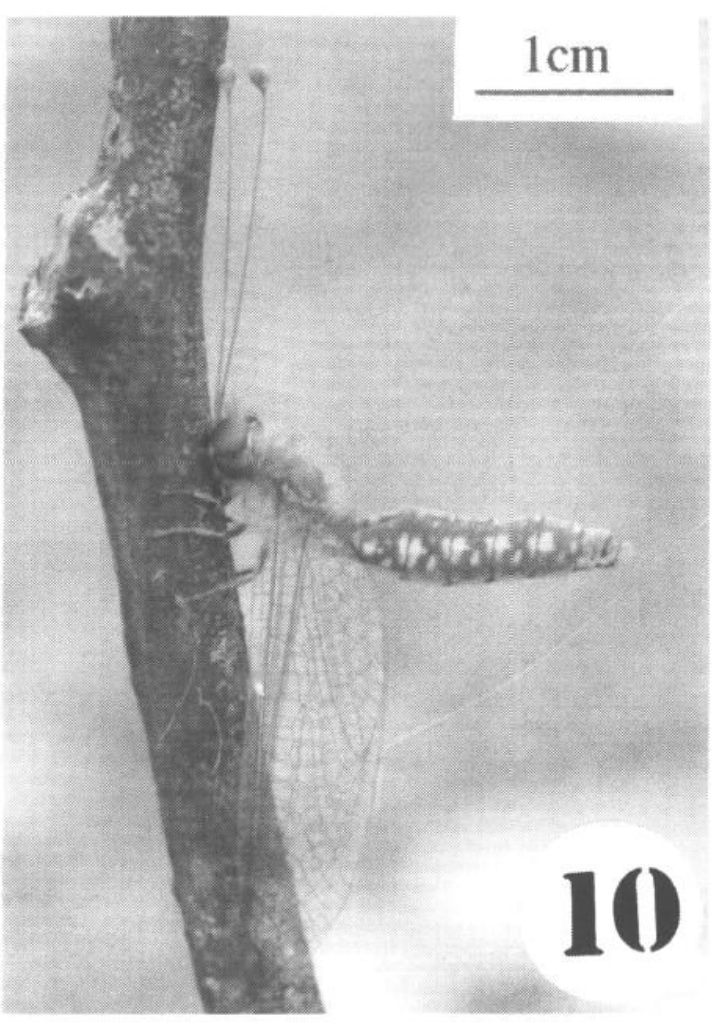

Fig. 10. - Tytomyia flinti Tjeder, $q$ au repos.
Antennes brunes avec les trois premiers articles plus clairs. Massues plus claires à la base. Thorax noir dessus avec les côtés bruns. Face supérieure avec des soie noires courtes; côtés avec des soies blanches plus longues et plus denses. Stigma avec trois nervures. Pattes jaunâtres avec la face inférieure des articles brun foncé à noir. Abdomen noir avec les tergites et les sternites bordés postérieurement de jaune. Sur le vivant les pleures sont tachées de blanc, de jaune clair et de gris (fig. 10). Ectoproctes arrondis, bordés extérieurement de jaune. Distivalves jaunes avec une tache basale noire. Ventrovalves noires avec la marge apicale bordée de jaune. Linguella jaune, non divisée, avec seulement une légère dépression longitudinale (fig. 8 et 9).

T. flinti fréquente les mêmes biotopes que C. kumari. C'est une espèce vive qui s'envole très facilement. Au repos la femelle relève son abdomen à $85^{\circ}$. La face est appliquée contre le support. Les antennes sont dressées avec les massues piriformes non repliées. Les ailes sont dirigées vers le bas (fig. 10). Le mâle adopte la même position.

\section{5 - Farakosius chloeae Michel, 1998}

Captures postérieures à la description de l'espèce. - Chutes de Farako: 7.VII.98, $12 \mathrm{~h}, 1$ \% ; 10.VII.98, 16 h 30,$19 ; 8 . I X .98$, entre $11 \mathrm{~h} 30$ et $12 \mathrm{~h}, 10$, 1 \% ; 28.X.97, 1 \% ; 11.XI.98, entre $16 \mathrm{~h}$ et $17 \mathrm{~h}, 1 \%$.

Capturé du 7 juillet au 11 novembre. Les récoltes de juillet avancent de près d'un mois le début de la période de vol par rapport aux informations présentées lors de la description de l'espèce (MICHEL, 1998).

\section{6 - Farakosius thaisae Michel, 1998}

Captures postérieures à la description de l'espèce. - Chutes de Farako: 23.X.97, 3 q ; 26.X.97, $1 \delta: 28 . X .97,3 q ; 11 . X I .98$, entre $16 \mathrm{~h}$ et $17 \mathrm{~h}, 20,1 \%$.

Capturé du 23 octobre au 8 décembre. Vole au début de la saison sèche. Sa période de vol est beaucoup plus courte que celle de $F$. chloeae.

\section{Discussion}

A. maculipennis, $P$. pardalis, $C$. kumari et $T$. flinti sont signalés pour la première fois du Mali. Les deux dernières espèces, considérées comme rares, sont représentées dans la région de Sikasso par des populations importantes mais peut-être isolées. Les deux Farakusius n'ont été observés qu'aux chutes de Farako (MiCHEL, 1998).

Suivant les espèces, la période de vol s'étend sur deux à cinq mois environ. $C$. kumari et $T$. flinti volent pendant la saison sèche. $F$. chloeae se rencontre pendant la période la plus humide de l'année. A. maculipennis, $P$. pardalis et $F$. thaisae apparaissent au cours des changements de saison (tableau I).

Comme pour les Myrmeleontidae (MICHEL, 1999), le sud du Mali, avec au moins douze espèces, héberge une faune d'Ascalaphes relativement riche. Outre les 6 espèces 
Tableau I. - Périodes de capture des Ascalaphidae dans le sud du Mali.

A.m.: Allocormodes maculipennis, P.p.: Phalascusa pardalis, C.k. : Cirrops kumari, T.f.: Tytomyia flinti, F.c.: Farakosius chloeae, F.t.: $F$. thaisae. $(\mathrm{m}=$ mâle; $\mathrm{f}=$ femelle).

\begin{tabular}{|c|c|c|c|c|c|c|c|c|c|c|c|c|}
\hline \multirow[t]{2}{*}{ esp. } & \multicolumn{12}{|c|}{ mois et décades } \\
\hline & janv. & fév. & mars & avr. & mai & juin & juill. & aoat & sep. & oct. & nov. & déc. \\
\hline A.m. $\mathrm{m}$ & & & & & & & & & & & & \\
\hline A.m. $\mathrm{f}$ & & & & & & & & & & & & \\
\hline P.p. $\mathrm{m}$ & & & & & & & & & & & & \\
\hline P.p f & & & & & & & & & & & & \\
\hline C.k. m & & & & & & & & & & & & \\
\hline C.k. $f$ & & & & & & & & & & & & \\
\hline T.f. $\mathrm{m}$ & & & & & & & & & & & & \\
\hline Tf. $\mathrm{f}$ & & & & & & & & & & & & \\
\hline F.C. $\mathrm{m}$ & & & & & & & & & & & & \\
\hline F.c. $f$ & & & & & & & & & & & & \\
\hline F.t. $\mathrm{m}$ & & & & & & & & & & & & \\
\hline F.t. $\mathrm{f}$ & & & & & & & & & & & & \\
\hline
\end{tabular}

mentionnées ci-dessus, les autres Ascalaphes que nous avons collectés appartiennent aux genres Ascalaphus ( 2 ou 3 espèces), Disparomitus (2 espèces), Stephanolasca (1 espèce) et ? Phalascusa (1 espèce). L'état actuel des connaissances sur ce groupe de Neuroptères ne permet malheureusement pas de les identifier avec certitude. De plus il est probable que ce matériel renferme au moins une espèce nouvelle.

REMERCIEMENTS. - Nos sincères remerciements au Dr A. Prost pour l'envoi des photographies des deux Allocormodes. Nous remercions également le Dr M. W. Mansell (Plant Protection Research Institute, Pretoria, Afrique du Sud) pour son accueil et sa disponibilité lors de notre passage à Johannesburg.

\section{AUTEURS CITÉS}

FRASER F.C., 1953. - Contribution à l'étude de la zone d'inondation du Niger (mission G. Remaudière 1950). I. - Odonata, Neuroptera and Mecoptera. Bulletin de l'Institut Français d'Afrique Noire, 15 (4) : $1523-1528$.

HYND W.R. B., 1992. - New items in Neuropteran distribution (Insecta: Neuroptera: Berothidae, Dilaridae, Ascalaphidae, Myrmeleontidae). Current Research in Neuropterology. Proceeding of the 4th International Symposium on Neuropterology, 24-27 juin 1991, Bagnères-de-Luchon, France : 189-192.

MiCHEL B., 1998. - Description d'un nouveau genre et de deux nouvelles espèces d'Ascalaphes afrotropicaux (Neuroptera, Ascalaphidae). Bulletin de la Société entomologique de France, 103 (1): 45-50.

— 1999. - Biodiversité et écologie des Palparinae du sud du Mali (Neuroptera, Myrmeleontidae). Bulletin de la Société entomologique de France, 104 (1): 45-52.

TJEDER B., 1980. - Ascalaphidae (Neuroptera) from Senegal and the Gambia. Entomologica scandinavica, $11: 401-412$.

1992. - The Ascalaphidae of the Afrotropical Region (Neuroptera). 1. External morphology and bionomics of the family Ascalaphidae, and taxonomy of the subfamily Haplogleniinae including the tribes Proctolyrini n. tribe, Melambrotini n. tribe, Campylophlebini n. tribe, Tmesibasini n. tribe, Allocormodini n. tribe, and Ululomyiini n. tribe of Ascalaphinae. Entomologica scandinavica, suppl., 41 : 1-169.

TJeder B. \& HANSSON C., 1992. - The Ascalaphidae of the Afrotropical Region (Neuroptera). 2. Revision of the tribe Ascalaphini (subfam. Ascalaphinae) excluding the genus Ascalaphus Fabricius. Entomologica scandinavica, suppl., 41 : 170-237. 